\title{
MicroRNA-150 suppresses the growth and malignant behavior of papillary thyroid carcinoma cells via downregulation of MUC4
}

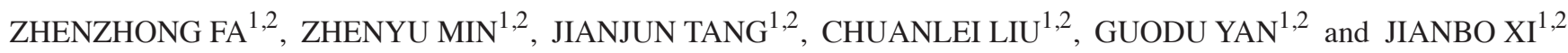 \\ ${ }^{1}$ Department of General Surgery, Wujin Affiliated Hospital of Jiangsu University; \\ ${ }^{2}$ Department of General Surgery, Changzhou Wujin People's Hospital, Changzhou, Jiangsu 213002, P.R. China
}

Received July 6, 2017; Accepted November 29, 2017

DOI: $10.3892 /$ etm.2018.6197

\begin{abstract}
Previous studies have revealed that microRNA (miR)-150 can act as an oncomiR or a tumor suppressor in numerous types of hematological malignancy and solid tumor. However, the function of miR-150 in papillary thyroid carcinoma (PTC) remains elusive. The present study aimed to investigate the function of miR-150 in PTC and its underlying molecular mechanism. The expression of miR-150 was identified to be significantly downregulated, whereas that of mucin (MUC)4 was significantly upregulated in PTC tissues and cell lines compared with corresponding controls. Further experiments demonstrated that MUC4 is a direct target of miR-150. PTC cell proliferation and capacity for migration and invasion decreased following miR-150 overexpression. It was also demonstrated that miR-150-mediated MUC4 downregulation was associated with an accompanying decrease in human epidermal growth factor receptor 2, as well as its phosphorylated form, resulting in suppressed activation of downstream signaling. In conclusion, the present study demonstrated that miR-150 may serve a key function in suppressing the malignant growth and aggressive behavior of PTC cells through the downregulation of MUC4. These findings may provide a novel approach for diagnostic and therapeutic strategies for PTC.
\end{abstract}

\section{Introduction}

Thyroid cancer, which represents $2 \%$ of all malignant disease cases and almost $90 \%$ of neuroendocrine cancer cases, is the most common endocrine malignant tumor (1,2). Papillary thyroid carcinoma (PTC) is the most common thyroid malignancy, with a faster increase in incidence over the past

Correspondence to: Dr Jianbo Xi, Department of General Surgery, Wujin Affiliated Hospital of Jiangsu University, 2 Yongning North Road, Changzhou, Jiangsu 213002, P.R. China

E-mail: dr_xijianbo@outlook.com

Key words: microRNA-150, mucin 4, papillary thyroid carcinoma, human epidermal growth factor receptor 2, focal adhesion kinase/extracellular signal-regulated kinase three decades $(3,4)$. The survival rate of patients with PTC is considered to be relatively favorable following complete thyroidectomy and treatment with levothyroxine and radioactive iodine (5). However, $10-15 \%$ of patients with PTC develop recurrence and distant metastases $(6,7)$. Therefore, there is a need for improved therapeutic strategies for PTC. Previous studies have implicated genetics in the pathogenesis of PTC, including activation of oncogenes and silencing of tumor-suppressor genes $(8,9)$. However, more efforts are required to identify key molecules that may be involved in the development of PTC and that may serve as potential therapeutic targets.

MicroRNAs (miRNAs) are a series of small non-coding RNAs, 19-25 nucleotides in length (10), that regulate post-transcriptional gene expression by binding to the 3'-untranslated region (3'-UTR) of target mRNAs (11). miRNAs serve key functions in cell proliferation, apoptosis and other biological processes in cancer (12). Aberrant expression and dysregulation of miRNAs has been observed in patients with pancreatic cancer (PC), as well as in cancer cell lines (8,13-15), indicating the potential value of miRNA profiles as cancer biomarkers and in the development of novel drugs.

miRNA (miR)-150 was first identified due to its crucial regulatory role in normal hematopoiesis (16). However, in recent years, researchers have demonstrated that aberrant expression and dysregulation of miR-150 is closely associated with various types of hematological malignancy (17) and solid tumor (18). miR-150 may act as an oncomiR or a tumor suppressor, depending on the targeted mRNA (17). Srivastava et al (19) observed downregulation of miR-150 in malignant pancreatic tissues and demonstrated the role of miR-150 in the regulation of mucin (MUC) 4 and tumor suppression in PC. The authors hypothesized that restoring miR-150 levels may be of therapeutic value in PC. Wu et al (20) revealed that miR-150 accelerated the spread of gastric cancer by downregulating the pro-apoptotic gene, early growth response 2 . In addition, Wang et al (21) highlighted a novel function for cyclin-dependent kinase 3 (CDK3) in myoblast cell proliferation and confirmed CDK3 as a key target that further enhances the tumor suppressor function of miR-150. However, the expression profile of miR-150 and its direct target in PTC remain elusive.

Based on previous reports (19-21), it was hypothesized that miR-150 may be differentially expressed in PTC and associated with the biological functions of PTC cells. Therefore, 
in the present study, the miR-150 expression profile was evaluated in PTC tissues and cell lines through reverse transcription-quantitative polymerase chain reaction (RT-qPCR) and western blotting. Through bioinformatics analysis, the potential targets of miR-150 were identified and the results were further confirmed by luciferase reporter assay. Cell viability, migration and invasion rates were also investigated in PTC cell lines.

\section{Materials and methods}

Cell lines and thyroid tissue specimens. The human PTC cell line TPC-1 and the normal thyroid cell line Nthy-ori 3-1 were purchased from (Sigma-Aldrich; Merck KGaA, Darmstadt, Germany). The cells were cultured and maintained in RPMI-1640 medium (Invitrogen; Thermo Fisher Scientific, Inc., Waltham, MA, USA) with $10 \%$ fetal bovine serum (FBS; Gibco; Thermo Fisher Scientific, Inc.) and penicillin-streptomycin (1:100; Sigma-Aldrich; Merck KGaA) according to a previous study (22) in an incubator with $5 \% \mathrm{CO}_{2}$ at $37^{\circ} \mathrm{C}$.

Thyroid tumor tissue and adjacent normal thyroid tissue samples were obtained from 30 patients (age range, 34-65 years; median age, 46; 12 males and 18 females) with PTC from May 2015 to July 2016 at Wujin Affiliated Hospital of Jiangsu University (Changzhou, China). All experiments involving human tissues were reviewed and approved by the Committee for Ethical Review of Research Involving Human Subjects at Wujin Affiliated Hospital of Jiangsu University. All patients provided written informed consent for the use of their tissues.

Cell transfection. miR-150 mimics (5'-UCUCCCAACCCU UGUACCAGUG-3') and negative control miR sequences (5'-CCGAAACCUCGGUUGAUUGCGG-3') were purchased from Shanghai GenePharma Co., Ltd. (Shanghai, China). Lipofectamine 2000 (Invitrogen; Thermo Fisher Scientific, Inc.) was used to perform TPC-1 cell transfection, according to the manufacturer's protocol. The cells were then cultured for $24 \mathrm{~h}$ at $37^{\circ} \mathrm{C}$ and $5 \% \mathrm{CO}_{2}$ for further analysis.

MTT assay. An MTT assay kit (Beyotime Institute of Biotechnology, Shanghai, China) was used to measure TPC-1 cell viability at 24,48 and $72 \mathrm{~h}$ after transfection, according to the manufacturer's protocol. TPC- 1 cells $\left(5 \times 10^{4}\right.$ per well) were cultured in 96-well plates and incubated for 24, 48 and $72 \mathrm{~h}$ at $37^{\circ} \mathrm{C}$. A total of $10 \mu 1 \mathrm{MTT}$ in PBS $(5 \mathrm{mg} / \mathrm{ml})$ was then added to each well and incubated at $37^{\circ} \mathrm{C}$ for $4 \mathrm{~h}$. Subsequently, the medium was removed and formazan crystals were dissolved using dimethyl sulfoxide $(150 \mu \mathrm{l} /$ well $)$ for $30 \mathrm{~min}$ at $37^{\circ} \mathrm{C}$. The absorbance was measured at a wavelength of $450 \mathrm{~nm}$, using a Bio-Rad iMark plate reader (Bio-Rad Laboratories, Inc., Hercules, CA, USA).

Cell migration and invasion assays. Wound healing and Transwell invasion experiments were used to evaluate cell migration and invasion, respectively. For the wound-healing assay, confluent monolayers of TPC-1 cells cultured in 24-well plates were mechanically wounded using a $10-\mu 1$ pipette tip. The wells were washed to remove cellular debris and the cells were allowed to migrate for $24 \mathrm{~h}$. Representative images were captured at x100 magnification under an inverted microscope (Olympus Corporation, Tokyo, Japan). The experiments were repeated at least three times. This assay was performed $24 \mathrm{~h}$ after transfection.

ForTranswellinvasion experiments,TPC-1 cells werecultured in $200 \mu \mathrm{l}$ RPMI-1640 medium in suspension $\left(5 \times 10^{5}\right.$ cells $\left./ \mathrm{ml}\right)$ and seeded into the upper chamber of a Transwell insert with an $8-\mathrm{mm}$ pore size membrane and a Matrigel-coated membrane matrix. RPMI-1640 medium with $10 \%$ FBS was added to the lower chamber as a chemoattractant. After incubation for $24 \mathrm{~h}$, non-migrated cells were removed with a cotton swab in the upper chamber of the Transwell insert and the migrated cells on the underside of the filter membrane were fixed in $100 \%$ methanol for $15 \mathrm{~min}$ at room temperature and stained with $0.1 \%$ crystal violet for $30 \mathrm{~min}$ at $37^{\circ} \mathrm{C}$ (Sigma-Aldrich; Merck KGaA) The migrated cells were counted and photographed (x100 magnification) in five randomly selected microscopic fields under an inverted microscope.

$R N A$ isolation and $R T-q P C R$. TRIzol reagent (Invitrogen; Thermo Fisher Scientific, Inc.) was used to extract total RNA from TPC-1 cells and thyroid tumor tissue and was then converted into cDNA using a Reverse Transcription kit (Thermo Fisher Scientific, Inc.) according to the manufacturer's protocol. The expression of miR-150 was determined with the bulge-loop ${ }^{\mathrm{TM}}$ miRNA qPCR Primer Set (Guangzhou RiboBio Co., Ltd., Guangzhou, China) and a SYBR-Green qPCR kit (Takara Biotechnology Co., Ltd., Dalian, China). U6 served as an internal control. The expression of MUC4 mRNA was analyzed by qPCR with the SYBR Premix Ex Taq ${ }^{\mathrm{TM}}$ kit (Takara Biotechnology, Co., Ltd.). GAPDH was used as an endogenous control. The primers for qPCR were obtained from GenScript (Piscataway, NJ, USA). The primer sequences as follows: MUC4, forward: 5'-GGACCAGAGCGAAAG CATTTGCC-3', reverse: 5'-TCAATCTCGGGTGGCTGA ACGC-3'; GAPDH, forward: 5'-CTGGGCTACACTGAG CACC-3', reverse: 5'-AAGTGGTCGTTGAGGGCAATG-3'; miR-150, forward: 5'-CTCAACTGGTGTCGTGGAGTC GGCAATTCAGTTGAGCACTGGTA-3', reverse: 5'-ACA CTCCAGCTGGGTCTCCCAACCCTTGTA-3'; U6, forward: 5'-CTCGCTTCGGCAGCACA-3', Reverse: 5'-AACGCT TCACGAATTTGCGT-3'. All experiments were performed according to the manufacturer's protocols. The thermocycling conditions for qPCR were as follows: $95^{\circ} \mathrm{C}$ for $5 \mathrm{~min}$, followed by 40 cycles of denaturation at $95^{\circ} \mathrm{C}$ for $15 \mathrm{sec}$ and annealing/elongation at $60^{\circ} \mathrm{C}$ for $30 \mathrm{sec}$. The $2^{-\Delta \Delta \mathrm{Cq}}$ method (23) was used to calculate the relative quantities of each gene. Data were obtained from three independent experiments.

Western blot analysis. Total protein was extracted from TPC-1 cells using RIPA lysis buffer (Thermo Fisher Scientific, Inc.) and then quantified using a BCA protein assay kit (Beyotime Institute of Biotechnology) Total protein (50 $\mu \mathrm{g} /$ lane) was then separated on $10 \%$ SDS-PAGE and transferred to polyvinylidene fluoride membranes (EMD Millipore, Billerica, MA, USA). The membranes were then blocked with $5 \%$ skimmed milk for $2 \mathrm{~h}$ and incubated overnight at $4{ }^{\circ} \mathrm{C}$ with the following primary antibodies at 1:1,000 dilution: Anti-MUC4 (catalog no. ab60720), anti-human epidermal growth factor receptor 2 (HER2; catalog no. ab16901), anti-p-HER2 (catalog no. ab53290), anti-focal 
adhesion kinase (FAK; catalog no. ab40794), anti-p-FAK (catalog no. ab81298), anti-extracellular signal-regulated kinase (ERK; catalog no. ab196883), anti-p-ERK (catalog no. ab4819), anti-GAPDH (catalog no. ab8245) (all Abcam, Cambridge, UK). The membranes were then washed and incubated with horseradish peroxidase-conjugated secondary antibodies (catalog nos. ab191866 and ab218695; dilution rate, 1:1,000; Abcam) for $1 \mathrm{~h}$ at room temperature. Immunoreactive bands were detected using the ChemiDoc XRS+ system (Bio-Rad Laboratories, Inc.) and an ECL Plus kit (Beyotime Institute of Biotechnology). Data were analyzed by densitometry using Image Pro Plus v.6.0 software (Media Cybernetics, Inc., Rockville, MD, USA). GAPDH served as an internal control for all experiments.

Bioinformatics prediction and Dual-luciferase reporter assay. Targetscan (www.targetscan.org/vert_71) and miRWalk (http://zmf.umm.uni-heidelberg.de/apps/ $\mathrm{zmf} / \mathrm{mirwalk} / \mathrm{micrornapredictedtarget.html)}$ were used to predicate the putative target genes of miR-150. To investigate the direct effect of miR-150 on the expression of MUC4, a miRNA target luciferase reporter assay was performed using a pEZX-MT01 target reporter plasmid containing the MUC4 3'-UTR region (GeneCopoeia, Inc., Rockville, MD, USA). Additionally, a mutant MUC4 3' UTR (MUT-MUC4 3' UTR) reporter construct was generated by site-directed mutagenesis in the putative target site of miR-150 in the wild-type (WT) MUC4 3'-UTR using the Quickchange XL site-directed mutagenesis kit (Agilent Technologies, Inc., Santa Clara, CA, USA). The reporter plasmids were co-transfected into cells with miR-150 mimics or the control vector using Lipofectamine 2000 (Invitrogen; Thermo Fisher Scientific, Inc.) in 24-well plates. The Dual-Luciferase Reporter Assay system (Promega Corporation, Madison, WI, USA) was used to perform luciferase activity $48 \mathrm{~h}$ following transfection, according to the manufacturer's protocol. Renilla luciferase activity was normalized to firefly luciferase activity. The results were obtained from three independent experiments.

Statistical analysis. All statistical analyses were performed using SPSS 19.0 software (IBM Corp., Armonk, NY, USA). Data are presented as the mean \pm standard deviation. Differences between groups were analyzed using the two-tailed Student's t-test. $\mathrm{P}<0.05$ was considered to indicate a statistically significant difference.

\section{Results}

Downregulation of miR-150 and upregulation of MUC4 in $P T C$. The expression level of miR-150 in PTC specimens and adjacent normal thyroid tissues was initially determined by RT-qPCR analysis. The results revealed that miR-150 levels in PTC were significantly lower compared with those in matched normal tissues (Fig. 1A). The expression level of MUC4 in PTC specimens and adjacent normal thyroid tissues was also determined and the results suggested that MUC4 was significantly upregulated in PTC compared with adjacent normal tissues (Fig. 1B-D). The expression patterns of miRNA-150 in TPC-1 human PTC cells and the normal thyroid cell line Nthy-ori 3-1 were analyzed to confirm the aforementioned findings. As indicated in Fig. 2A, the expression of miR-150 was significantly downregulated in PTC cells. The expression level of MUC4 in PTC cells and normal thyroid cells was then measured by RT-qPCR and western blotting. The mRNA and protein levels of MUC4 were significantly higher in PTC cells compared with normal thyroid cells (Fig. 2B-D).

Upregulation of miR-150 suppresses human PTC cell proliferation and metastasis. To investigate the biological functions of miR-150 in the proliferation and invasion of human PTC cells, which are closely associated with tumorigenesis, gain-of function experiments were performed by transfection of miR-150 mimics in TPC- 1 cells. The level of miR-150 in the treated cells was assessed by RT-qPCR. The results revealed that the expression level of miR-150 was significantly increased in cells transfected with miR-150 mimics compared with the negative control (Fig. 3A). This result suggested that the transfection of miR-150 mimics in TPC-1 cells was successful. Thus, in the following experiments, the level of miR-150 was manipulated by transfection with miR-150 mimics, in order to investigate the potential role of miR-150 in the proliferation and invasion of human PTC cells.

The effect of miR-150 on cell proliferation was examined using an MTT assay. The results demonstrated that upregulation of miR-150 significantly inhibited cell proliferation at $72 \mathrm{~h}$ following transfection with the miR-150 mimic compared with the control group (Fig. 3B). This is important, since metastasis in late-stage disease adds to the difficulties of cancer treatment (24). Thus, wound-healing and Transwell invasion assays were performed to evaluate the effect of miR-150 on cell migration and invasion, respectively. As indicated in Fig. 3C-F, upregulation of miR-150 significantly suppressed cancer cell migration and invasion.

MUC4 is a direct target of miR-150 in PTC cells. Through bioinformatics analysis via the Targetscan (www.targetscan. org/vert_71) and miRWalk (http://zmf.umm.uni-heidelberg. de/apps/zmf/mirwalk/micrornapredictedtarget.html) websites, MUC4 was identified to be a potential target of miR-150. As indicated in Fig. 4A, a putative binding site for miR-150 was identified in MUC4 3'-UTR. To determine whether MUC4 expression was mediated by miR-150, a dual-luciferase reporter assay was performed. The 3'-UTR of MUC4 mRNA, including the putative miR-150 binding sequence (WT 3'-UTR) or the mutant sequence (MUT 3'-UTR), was subcloned into luciferase reporter plasmids. TPC-1 cells were then co-transfected with the WT or MUT 3'-UTR of MUC4 and miR-150 mimics or controls. The luciferase results indicated that overexpression of miR-150 in TPC-1 cells co-transfected with MUC4 WT led to a significant reduction of luciferase activity, while there was no such reduction in the TPC-1 cells co-transfected with MUC4 MUT (Fig. 4B). These results suggested that MUC4 was the direct target of miR-150. The luciferase activity of miR-150-transfected cells was markedly decreased compared with the controls (Fig. 4B). The effects of miR-150 upregulation on MUC4 mRNA and protein expression in TPC-1 cells were also analyzed. Compared with cells transfected with negative control sequences, cells transfected with miR-150 mimics exhibited significantly decreased mRNA and protein levels of MUC4 (Fig. 4C-E). 
A
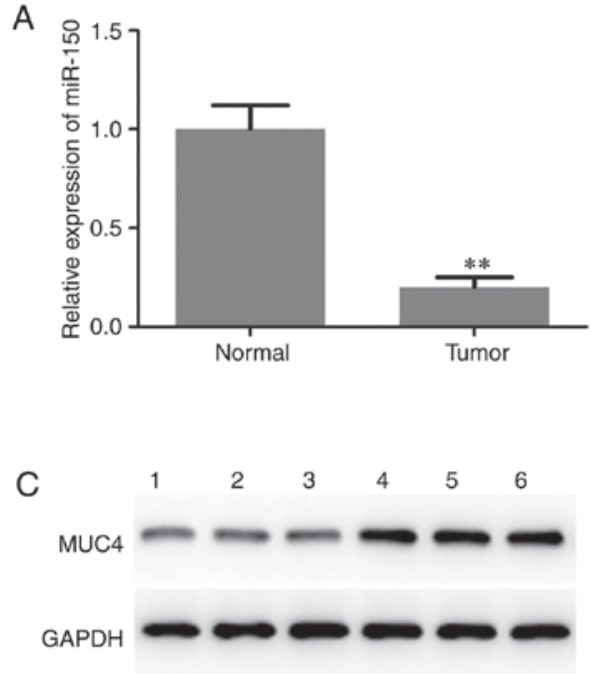

B

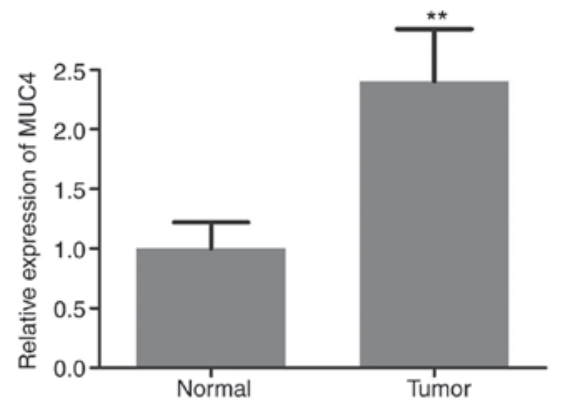

D

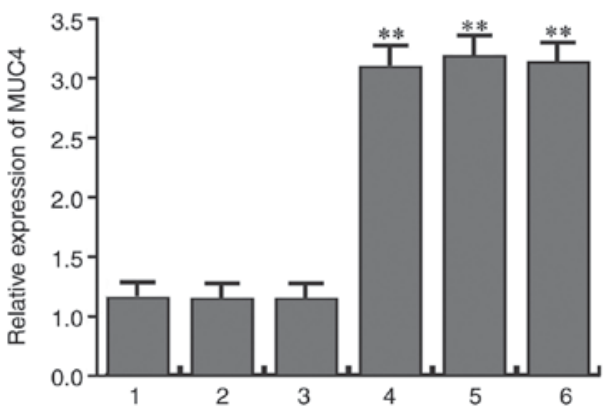

Figure 1. Downregulation of miR-150 and upregulation of MUC4 in PTC tissues. (A) Quantitative analysis of miR-150 levels in PTC and adjacent normal tissues as determined by RT-qPCR. (B) Quantitative analysis of MUC4 levels in PTC and adjacent normal tissues as determined by RT-qPCR. (C) Protein expression level of MUC4 in PTC $(4,5,6)$ and adjacent matched normal tissues $(1,2,3)$ was determined by western blot assay. (D) Densitometric analysis of MUC4 protein level. " $\mathrm{P}<0.01$ vs. matched adjacent normal tissues. miR-150, microRNA-150; MUC4, mucin 4; PTC, papillary thyroid carcinoma; RT-qPCR, reverse transcription-quantitative polymerase chain reaction.

A

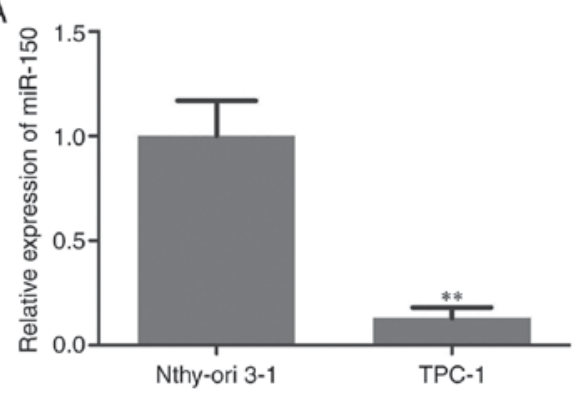

C

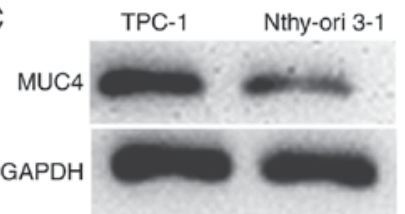

B

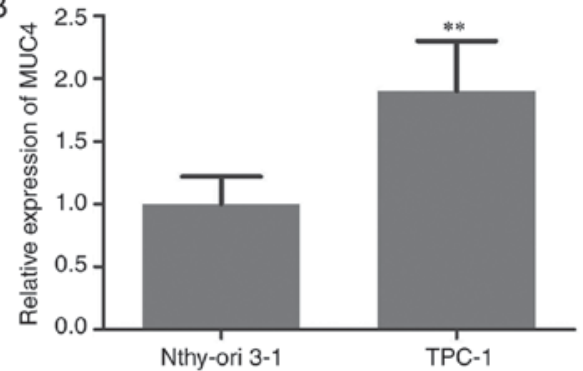

D

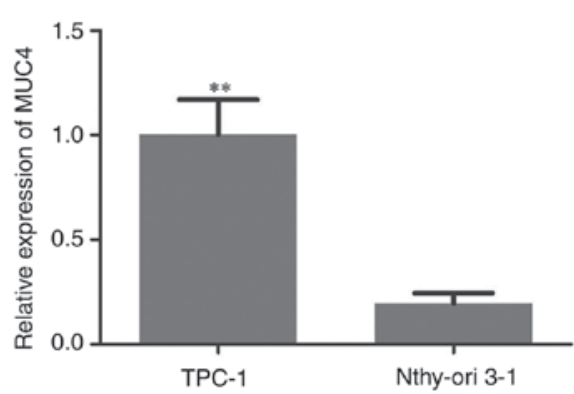

Figure 2. Downregulation of miR-150 and upregulation of MUC4 in papillary thyroid carcinoma cells. (A) Relative miR-150 expression in TPC-1 and Nthy-ori 3-1 cell lines. (B) mRNA and (C) protein expression level of MUC4 in TPC-1 and Nthy-ori 3-1 cell lines, as determined by reverse transcription-quantitative polymerase chain reaction and western blot analysis. (D) Densitometric analysis of MUC4 protein level. ${ }^{* *} \mathrm{P}<0.01$ vs. Nthy-ori 3-1 cells. miR-150, microRNA-150; MUC4, mucin 4.

Effect of miR-150 on HER2 and FAK/ERK downstream signaling. To elucidate the molecular mechanism of miR-150, HER2, an interacting partner of MUC4 and downstream FAK/ERK signaling were investigated in TPC-1 cells. As indicated in Fig. 5, the expression of HER2 and p-HER2 was significantly decreased following transfection of miR-150 mimics in TPC-1 cells as compared with the control. A significant decrease in p-FAK and p-ERK was also observed in miR-150-transfected cells compared with the control, whereas no significant changes in the expression levels of total FAK and ERK were observed. The results suggested a critical role for MUC4 in the expression of HER2 and p-HER2 and the phosphorylation of FAK and ERK, key cancer survival molecules. In addition, the activation of HER2 and FAK/ ERK signaling 

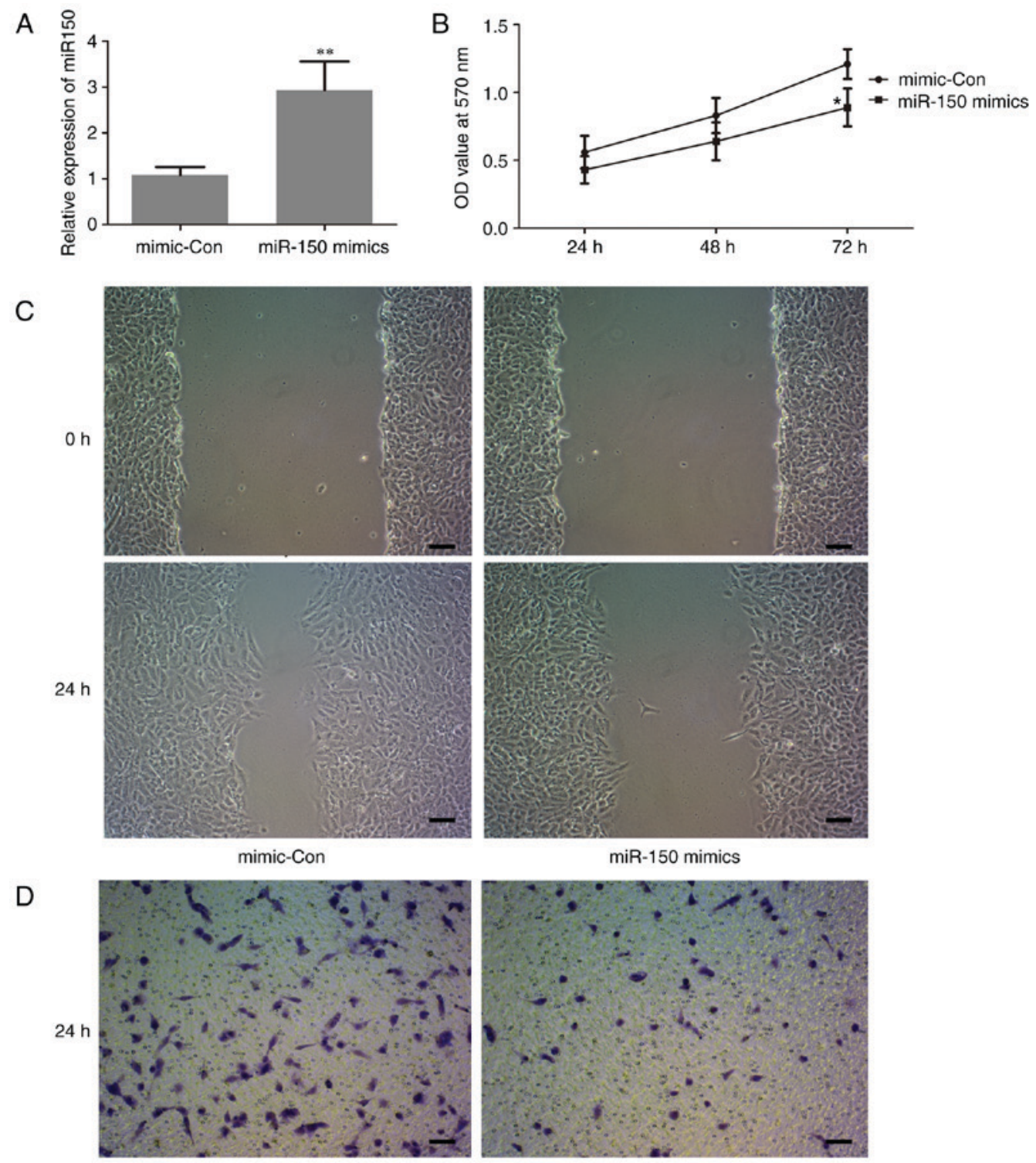

mimic-Con

miR-150 mimics
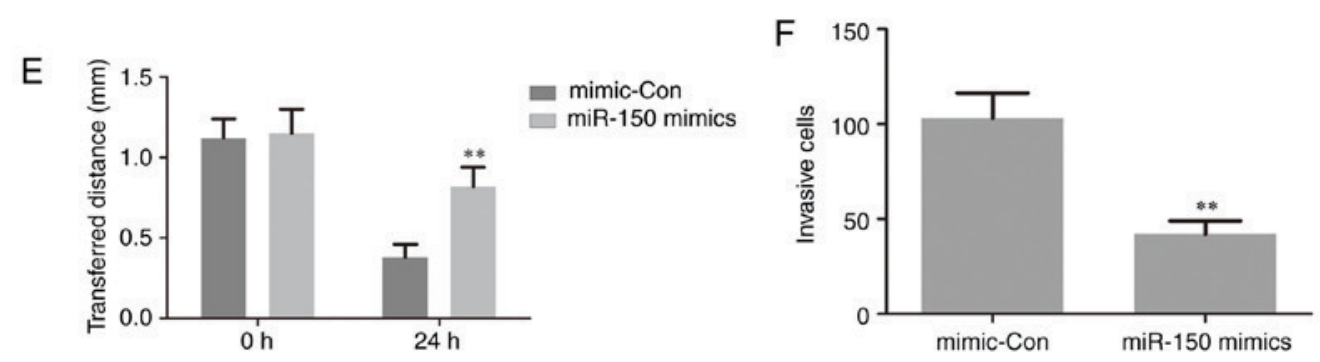

Figure 3. Upregulation of miR-150 suppresses human papillary thyroid carcinoma cell proliferation and metastasis. (A) Relative miR-150 expression in TPC-1 cells following transfection. (B) Proliferation of TPC-1 cells following transfection. (C) Representative micrographs of a wound-healing assay in TPC-1 cells following transfection (Scale bars=50 $\mu \mathrm{m}$ ). (D) Representative micrographs of Transwell invasion experiments in TPC-1 cells following transfection (Scale bars $=50 \mu \mathrm{m}$ ). (E) Statistical analysis of the wound-healing assay results. (F) Statistical analysis of the Transwell invasion assay results. "P<0.05 vs. mimic-Con, ${ }^{* *} \mathrm{P}<0.01$ vs. mimic-Con. miR-150, microRNA-150; mimic-Con, negative control transfection; OD, optical density.

was indicated to be attenuated by the upregulation of miR-150 in PTC.

\section{Discussion}

miRNAs not only serve critical functions in regulating numerous fundamental cellular processes, but may also act as oncogenes or tumor suppressor genes in a variety of human cancer types, including PTC (25). Over the past few years, an increasing number of studies have been focused on the role of miRNA expression in PTC $(26,27)$. It has been reported that miRNA dysregulation is associated with pathological processes in PTC, as reflected by the extent of extrathyroidal invasion, tumor size and the presence of lymph node metastases (8). miRNA-146, 

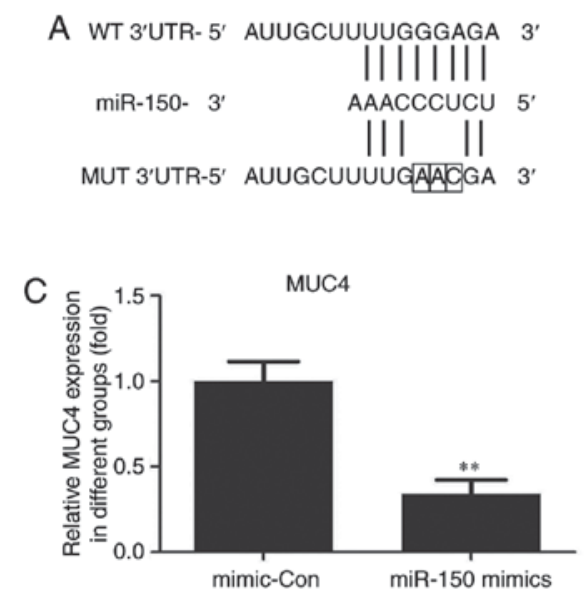
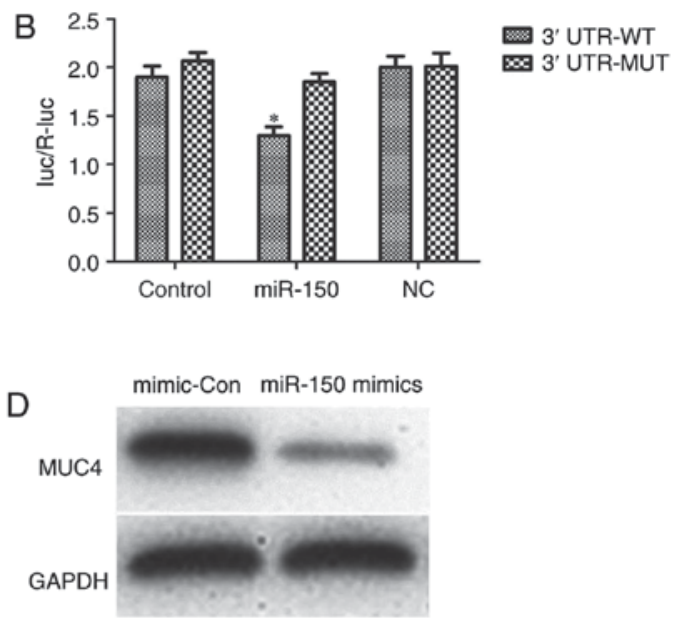

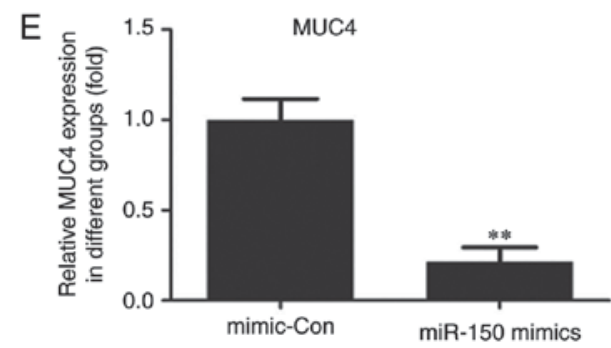

Figure 4. MUC4 is a direct target of miR-150 in papillary thyroid carcinoma cells. (A) miR-150 and its target site in MUC4 3'-UTR. The mutant bases are indicated by boxes. (B) Luciferase reporter assay in TPC-1 cells transfected with reporter vectors containing WT or MUT MUC4 3'-UTR. (C) MUC4 mRNA expression was evaluated by reverse transcription-quantitative polymerase chain reaction in TPC-1 cells transfected with miR-150 mimics and mimic-Con. (D) MUC4 protein expression was evaluated by western blot analysis in TPC-1 cells transfected with miR-150 mimics and mimic-Con. (E) Densitometric analysis of MUC4 protein level. ${ }^{*} \mathrm{P}<0.05$ vs. control; ${ }^{* *} \mathrm{P}<0.01$ vs. mimic-Con. miR-150, microRNA-150; mimic-Con, negative control transfection; MUC4, mucin 4; 3'-UTR, 3'-untranslated region; NC, negative control; WT, wild type; MUT, mutated.

-222 and -221 are the most common miRNAs involved in these processes and they are closely associated with tumor cell proliferation, differentiation, migration and apoptosis (8). Clearly identifying the unique expression profiles of cancer-specific miRNAs and their targets is crucial for elucidating their role in tumorigenesis and miRNAs have demonstrated potential in the development of cancer biomarkers and novel therapeutic drugs (8). It has been reported that miR-150 is up- or downregulated in a variety of solid cancer types, including cervical, breast, lung, colorectal, gastric, liver, pancreatic and esophageal cancer (19-21,28-30). However, the role of miR-150 in human PTC has not been fully elucidated.

In the present study, it was observed that miR-150 was decreased in PTC tissues and downregulated in human PTC cell lines. Thus, PTC cells were transfected with miR-150 mimics to upregulate the expression of miR-150. The transfection rate was satisfactory, which was confirmed by RT-qPCR assay. The biological effect of miR-150 on human PTC cells was then examined and the results demonstrated that overexpression of miR-150 significantly suppressed human PTC cell proliferation at $72 \mathrm{~h}$ following transfection with the miR-150 mimic. In addition, the overexpression of miR-150 significantly suppressed human PTC cell metastasis. Further experiments were performed to determine the potential underlying molecular mechanism of miR-150.

By means of bioinformatics analysis, a search for target genes of miR-150 was performed and MUC4 was identified as a potential target. MUC4 is a high molecular weight type I transmembrane protein and alterations of MUC4 are often associated with carcinomas (31). Upregulation of MUC4 has been demonstrated in several cancer types, including PTC (32). In a study of 98 patients by Nam et al (33), the gene expression of MUC4 increased by $\sim 78$-fold in PTC and the protein staining scores of MUC4 also increased markedly in PTC compared with normal thyroid tissue. The results indicated that high MUC4 expression was associated with small tumor size and the papillary thyroid microcarcinoma subtype. MUC4 may serve a key function in the early oncogenesis of PTC. However, the functional role of MUC4 in PTC and its dysregulation has not been clearly determined. In the present study, the mRNA and protein levels of MUC4 were identified to be significantly higher in PTC cells compared with normal thyroid cells, whereas miR-150 mimics significantly decreased the level of MUC4. Furthermore, the dual-luciferase reporter system suggested that MUC4 was the direct target of miR-150. Since there are numerous target genes of miR-150, rescue experiments are required to further investigate miR-150 function as a tumor suppressor via MUC4 in PTC. An in-depth study will be conducted in the future.

The majority of cancer-associated cases of mortality are attributed to metastasis (34). Cancer cell metastasis involves a complicated series of phenotypic and biochemical processes and events, including cell migration and invasion (35). FAK serves a vital function in tumor cell proliferation, survival and migration. FAK deficiency results in reduced cell motility and enhanced focal adhesion contact formation compared 

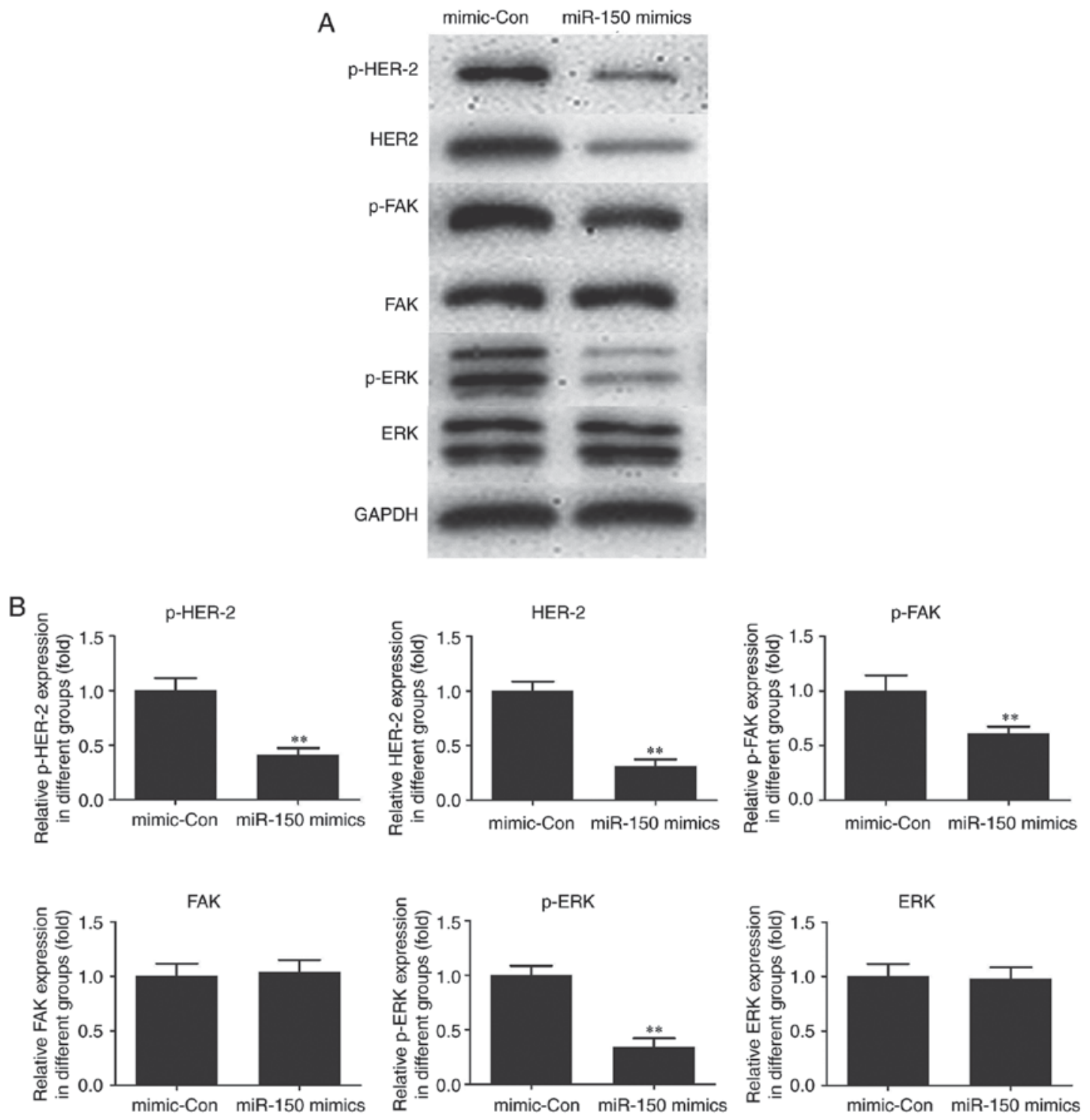

Figure 5. Effect of miR-150 on HER2 and downstream FAK/ERK signaling. (A) Protein levels were evaluated by western blot analysis. (B) Densitometric analysis of each protein level. ${ }^{* *} \mathrm{P}<0.01$ vs. mimic-Con. miR-150, microRNA-150; mimic-Con, negative control transfection; HER2, human epidermal growth factor receptor 2; FAK, focal adhesion kinase; ERK, extracellular signal-regulated kinase.

with control cells (36). FAK interactions with HER2 promote tumorigenesis and micrometastatic cells express activated/ p-FAK and HER2, suggesting a role for HER2-FAK activation in malignant and invasive growth (37). ERK is a member of the mitogen-activated protein kinase family that is involved in a broad range of cell functions and physiological processes (38). ERK is a well-known downstream signaling molecule of FAK and serves as a key regulatory component in cell motility (39). In the present study, decreased expression of HER2 and p-HER2 was identified in miR-150-overexpressing PTC cells. A similar decrease in p-FAK and p-ERK was also observed in miR-150-transfected cells, indicating that MUC4 serves a key role in the activation of these cancer-associated molecules and this effect may be partly modulated through the alteration of miR-150 in PTC.

In summary, the findings of the present revealed that miR-150 possesses antitumor properties and suppresses the growth and malignant behavior of PTC cells, at least in part via regulation of the downstream target MUC4. These findings may provide a novel insight into diagnostic and therapeutic strategies for PTC. However, the present study was limited by the fact that only one tumor cell line was investigated, thus in-depth studies will be conducted in the future in order to validate these results.

\section{Acknowledgements}

The authors are would like to thank Professor Caiping Huang of Head and Neck Surgery Department in Shanghai Cancer Hospital for his kind surgery advice. In addition, the authors appreciate the help provided by Dr Zhixin Xue of Changzhou Wujin People's Hospital in collecting tissue samples.

\section{References}

1. Xiang D, Xie L, Xu Y, Li Z, Hong Y and Wang P: Papillary thyroid microcarcinomas located at the middle part of the middle third of the thyroid gland correlates with the presence of neck metastasis. Surgery 157: 526-533, 2015. 
2. Kim HY, Park WY, Lee KE, Park WS, Chung YS, Cho SJ and Youn YK: Comparative analysis of gene expression profiles of papillary thyroid microcarcinoma and papillary thyroid carcinoma. J Cancer Res Ther 6: 452-457, 2010.

3. Torre LA, Bray F, Siegel RL, Ferlay J, Lortet-Tieulent J and Jemal A: Global cancer statistics, 2012. CA Cancer J Clin 65 : $87-108,2015$

4. Hughes DT, Haymart MR, Miller BS, Gauger PG and Doherty GM: The most commonly occurring papillary thyroid cancer in the United States is now a microcarcinoma in a patient older than 45 years. Thyroid 21: 231-236, 2011

5. Chou CK, Yang KD, Chou FF, Huang CC, Lan YW, Lee YF, Kang HY and Liu RT: Prognostic implications of miR-146b expression and its functional role in papillary thyroid carcinoma. J Clin Endocrinol Metab 98: E196-E205, 2013

6. Lee JC, Zhao JT, Clifton-Bligh RJ, Gill A, Gundara JS, Ip JC, Glover A, Sywak MS, Delbridge LW, Robinson BG and Sidhu SB: MicroRNA-222 and microRNA-146b are tissue and circulating biomarkers of recurrent papillary thyroid cancer. Cancer 119: 4358-4365, 2013

7. Lang BH, Tang AH, Wong KP, Shek TW, Wan KY and Lo CY: Significance of size of lymph node metastasis on postsurgical stimulated thyroglobulin levels after prophylactic unilateral central neck dissection in papillary thyroid carcinoma. Ann Surg Oncol 19: 3472-3478, 2012

8. Chruścik A and Lam AK: Clinical pathological impacts of microRNAs in papillary thyroid carcinoma: A crucial review. Exp Mol Pathol 99: 393-398, 2015.

9. Liang J, Cai W, Feng D, Teng H, Mao F, Jiang Y, Hu S, Li X, Zhang Y, Liu B and Sun ZS: Genetic landscape of papillary thyroid carcinoma in the Chinese population. J Pathol 244 215-226, 2018.

10. Hale BJ, Yang CX and Ross JW: Small RNA regulation of reproductive function. Mol Reprod Dev 81: 148-159, 2014.

11. Chi SW, Zang JB, Mele A and Darnell RB: Argonaute HITS-CLIP decodes microRNA-mRNA interaction maps. Nature 460: 479-486, 2009.

12. Wang XZ, Hang YK, Liu JB, Hou YQ, Wang N and Wang MJ: Over-expression of microRNA-375 inhibits papillary thyroid carcinoma cell proliferation and induces cell apoptosis by targeting ERBB2. J Pharmacol Sci 130: 78-84, 2016.

13. Borrelli N, Denaro M, Ugolini C, Poma AM, Miccoli M, Vitti P, Miccoli P and Basolo F: miRNA expression profiling of "noninvasive follicular thyroid neoplasms with papillary-like nuclear features' compared with adenomas and infiltrative follicular variants of papillary thyroid carcinomas. Mod Pathol 30: 39-51, 2017.

14. Ma X, Wei J, Zhang L, Deng D, Liu L, Mei X, He X and Tian J: miR-486-5p inhibits cell growth of papillary thyroid carcinoma by targeting fibrillin-1. Biomed Pharmacother 80: 220-226, 2016

15. Liu L, Wang J, Li X, Ma J, Shi C, Zhu H, Xi Q, Zhang J, Zhao X and Gu M: miR-204-5p suppresses cell proliferation by inhibiting IGFBP5 in papillary thyroid carcinoma. Biochem Biophys Res Commun 457: 621-626, 2015.

16. Vasilatou D, Papageorgiou S, Pappa V, Papageorgiou E and Dervenoulas J: The role of microRNAs in normal and malignant hematopoiesis. Eur J Haematol 84: 1-16, 2010.

17. He Y, Jiang $X$ and Chen J: The role of miR-150 in normal and malignant hematopoiesis. Oncogene 33: 3887-3893, 2014.

18. Wang F, Ren X and Zhang X: Role of microRNA-150 in solid tumors. Oncol Lett 10: 11-16, 2015.

19. Srivastava SK, Bhardwaj A, Singh S, Arora S, Wang B, Grizzle WE and Singh AP: MicroRNA-150 directly targets MUC4 and suppresses growth and malignant behavior of pancreatic cancer cells. Carcinogenesis 32: 1832-1839, 2011.

20. Wu Q, Jin H, Yang Z, Luo G, Lu Y, Li K, Ren G, Su T, Pan Y, Feng B, et al: miR-150 promotes gastric cancer proliferation by negatively regulating the pro-apoptotic gene EGR2. Biochem Biophys Res Commun 392: 340-345, 2010.

21. Wang L, Xi Y, Sun C, Zhang F, Jiang H, He Q and Li D: CDK3 is a major target of miR-150 in cell proliferation and anti-cancer effect. Exp Mol Pathol 102: 181-190, 2017.
22. Zhou D, Li Z and Bai X: BRAFV600E and RET/PTC promote proliferation and migration of papillary thyroid carcinoma cells in vitro by regulating nuclear factor- $\kappa \mathrm{B}$. Med Sci Monit 23 5321-5329, 2017

23. Livak KJ and Schmittgen TD: Analysis of relative gene expression data using real-time quantitative PCR and the 2(-Delta Delta $\mathrm{C}(\mathrm{T})$ ) method. Methods 25: 402-408, 2001

24. Talmadge JE and Fidler IJ: AACR centennial series: The biology of cancer metastasis: Historical perspective. Cancer Res 70: 5649-5669, 2010

25. Saiselet $M$, Gacquer D, Spinette A, Craciun L, Decaussin-Petrucci M, Andry G, Detours V and Maenhaut C: New global analysis of the microRNA transcriptome of primary tumors and lymph node metastases of papillary thyroid cancer. BMC Genomics 16: 828, 2015.

26. Qiu Z, Li H, Wang J and Sun C: miR-146a and miR-146b in the diagnosis and prognosis of papillary thyroid carcinoma. Oncol Rep 38: 2735-2740, 2017.

27. Kolanowska M,Wójcicka A,Kubiak A, Świerniak M, Kotlarek M, Maciąg M, Gaj P, Koperski Ł, Górnicka B and Jażdżewski K: Functional analysis of a novel, thyroglobulin-embedded microRNA gene deregulated in papillary thyroid carcinoma. Sci Rep 7: 9942, 2017

28. Li J, Hu L, Tian C, Lu F, Wu J and Liu L: microRNA-150 promotes cervical cancer cell growth and survival by targeting FOXO4. BMC Mol Biol 16: 24, 2015.

29. Huang S, Chen Y, Wu W, Ouyang N, Chen J, Li H, Liu X, Su F, Lin L and Yao Y: miR-150 promotes human breast cancer growth and malignant behavior by targeting the pro-apoptotic purinergic P2X7 receptor. PLoS One 8: e80707, 2013.

30. Cao M, Hou D, Liang H, Gong F, Wang Y, Yan X, Jiang X, Wang C, Zhang J, Zen K, et al: miR-150 promotes the proliferation and migration of lung cancer cells by targeting SRC kinase signalling inhibitor 1. Eur J Cancer 50: 1013-1024, 2014

31. Gautam SK, Kumar S, Cannon A, Hall B, Bhatia R, Nasser MW, Mahapatra S, Batra SK and Jain M: MUC4 mucin-a therapeutic target for pancreatic ductal adenocarcinoma. Expert Opin Ther Targets 21: 657-669, 2017.

32. Singh AP, Moniaux N, Chauhan SC, Meza JL and Batra SK: Inhibition of MUC4 expression suppresses pancreatic tumor cell growth and metastasis. Cancer Res 64: 622-630, 2004.

33. Nam KH, Noh TW, Chung SH, Lee SH, Lee MK, Hong SW, Chung WY, Lee EJ and Park CS: Expression of the membrane mucins MUC4 and MUC15, potential markers of malignancy and prognosis, in papillary thyroid carcinoma. Thyroid 21: 745-750, 2011.

34. I Valderrama-Treviño A, Barrera-Mera B, C Ceballos-Villalva J and E Montalvo-Javé E: Hepatic metastasis from colorectal cancer. Euroasian J Hepatogastroenterol 7: 166-175, 2017.

35. Sun J, Luo Q, Liu L, Yang X, Zhu S and Song G: Salinomycin attenuates liver cancer stem cell motility by enhancing cell stiffness and increasing F-actin formation via the FAK-ERK1/2 signalling pathway. Toxicology 384: 1-10, 2017.

36. Zhao $X$ and Guan JL: Focal adhesion kinase and its signaling pathways in cell migration and angiogenesis. Adv Drug Deliv Rev 63: 610-615, 2011.

37. Zhang J and Hochwald SN: The role of FAK in tumor metabolism and therapy. Pharmacol Ther 142: 154-163, 2014.

38. Mendoza MC, Vilela M, Juarez JE, Blenis J and Danuser G: ERK reinforces actin polymerization to power persistent edge protrusion during motility. Sci Signal 8: ra47, 2015.

39. Natarajan M, Hecker TP and Gladson CL: FAK signaling in anaplastic astrocytoma and glioblastoma tumors. Cancer $\mathbf{J}$ 9: 126-133, 2003

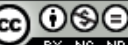

This work is licensed under a Creative Commons Attribution-NonCommercial-NoDerivatives 4.0 International (CC BY-NC-ND 4.0) License. 